\title{
Acknowledgement to Reviewers of Systems in 2016
}

\author{
Systems Editorial Office \\ Published: 11 January 2017 \\ MDPI AG, St. Alban-Anlage 66, 4052 Basel, Switzerland; systems@mdpi.com
}

The editors of Systems would like to express their sincere gratitude to the following reviewers for assessing manuscripts in 2016.

We greatly appreciate the contribution of expert reviewers, which is crucial to the journal's editorial process. We aim to recognize reviewer contributions through several mechanisms, of which the annual publication of reviewer names is one. Reviewers receive a voucher entitling them to a discount on their next MDPI publication and can download a certificate of recognition directly from our submission system. Additionally, reviewers can sign up to the service Publons (https://publons.com) to receive recognition. Of course, in these initiatives we are careful not to compromise reviewer confidentiality. Many reviewers see their work as a voluntary and often unseen part of their role as researchers. We are grateful to the time reviewers donate to our journals and the contribution they make.

If you are interested in becoming a reviewer for Systems, see the link at the bottom of the webpage http://www.mdpi.com/reviewers.

The following reviewed for Systems in 2016:

\begin{tabular}{lll} 
Ahn, Choon Ki & Divigalpitiya, Prasanna & Hellier, Alan Keith \\
Ardjmand, Ehsan & Doolan, Matthew & Herrmann, Frank \\
Arroyo, Liliana & Dorça, Fabiano Azevedo & Hoel, Tore \\
Azouani, Abderrahim & Esmaeili, Behzad & Hong, I-Hsuan \\
Bianca, Carlo & Fan, Chia-Ming & Hoornweg, Daniel \\
Blomqvist, Eva & Faravelli, Lucia & Houghton, Luke \\
Bontje, Marco & Feng, Tao & Hovmand, Peter S. \\
Borsi, Iacopo & Figueroa Meza, Maria Josefina & Hu, Bo \\
Cao, Jinde & Fink, Olga & Hvattum, Lars Magnus \\
Carbonara, Nunzia & Foley, Brian & Ishizaka, Alessio \\
Cartwright, Edward & Fortuna, Luigi & Jabłoński, Adam \\
Champion, Donna & Garcia-Cabot, Antonio & Jaradat, Raed M. \\
Chen, Lieu-hen & Gaudou, Benoit & Jin, Jian \\
Chen, Weiqin & Giuliani, Alessandro & Karagiannidis, Charalampos \\
Corkindale, David & González-Castolo, Juan C. & Khan, Khalid \\
Cornish-Bowden, Athel & Goyeneche, Dardo & Kim, Hyunjung \\
Cronin, Matthew & Grabski, Severin & Kitto, Kirsty \\
Daim, Tugrul & Graditi, Giorgio & Langford, Gary \\
Davidson, Colin & Guitouni, Adel & Larsen, Peter Gorm \\
De Carvalho, Rogerio Atem & Gunawan, Indra & Li, Dapeng \\
De Paola, Francesco & Haddara, Moutaz & Licata, Ignazio \\
Deakin, Mark & Hadzikadic, Mirsad & Lima, Vanda \\
Del Giudice, Manlio & Hamalainen, Raimo & Liu, Lei \\
Desmet, Piet & Han, Sekyung & Lizcano, David \\
Devine, Christopher & Harmon, Alison & Lopes, Myriam \\
Dickerson, Charles & Hawking, Paul & López, José Antonio \\
\hline
\end{tabular}




Love, Terence
Luo, Yugong
Luoma, Jukka
Ma, Huanfei
Machado, J. A. Tenreiro
Maditinos, Dimitrios I.
Mavrikis, Manolis
Menassa, Carol
Mohammadi, Arash
Monat, Jamie P.
Monje Micharet, Concepción Alicia
Mouter, Niek
Nelatury, Sudarshan R.
Nescolarde-Selva, Josué-Antonio
Newell, Barry
Nian, Xiaohong
Panagopoulos, Thomas
Papanikolaou, Kyparisia
Peñarrocha, Ignacio
Perc, Matjaz
Pohle, Ina
Polonsky, Michael
Pothos, Emmanuel
Prähofer, Herbert
Precup, Radu-Emil

\author{
Pryss, Rüdiger \\ Rada, Elena \\ Rauch, Erwin \\ Rennie, Frank \\ Rentrop, Christopher \\ Risco-Martín, José L. \\ Rogers, Eric \\ Rosen, Devan \\ Rousseau, David \\ Rubio-Campillo, Xavier \\ Saez Cornellana, Meritxell \\ Sampaio, Paulo \\ Sánchez García, Javier \\ Sandgren, Eric \\ Sarma, Santanu \\ Scherer, William T. \\ Seidl, Roman \\ Shang, Yilun \\ Shaw, Eric \\ Sireli, Yesim \\ Smith, Eric \\ Sohaib, Osama \\ Soibelman, Lucio \\ Solin, Jeremy \\ Sturiale, Luisa
}

$\mathrm{Su}$, Xiaojie

Sugano, Masashi

Sun, Shipeng

Svitek, Miroslav

Szolnoki, Attila

Tseng, Ming-Lang

Tudor, Terry

Ülkü, M. Ali

Ullah, Shatif

Uslar, Mathias

Uusitalo, Ville

Van Do, Tien

Venkatraman, Sitalakshmi

Verhagen, Philip

Wade, Jon

Wang, Chia-Yu

Wright, Steve

Yang, Ming

Yannis, George

Yearworth, Mike

Yoshimoto, Shusuke

Yukalov, Vyacheslav

Zambrano, Davide

(C) 2017 by the authors; licensee MDPI, Basel, Switzerland. This article is an open access article distributed under the terms and conditions of the Creative Commons Attribution (CC-BY) license (http://creativecommons.org/licenses/by/4.0/). 